\title{
2D SYNCHROTRON RADIATION INTERFEROMETER FOR MEASURING THE TRANSVERSE DIMENSIONS OF AN ELECTRON BEAM IN A CIRCULAR ACCELERATOR
}

\author{
A. I. Novokshonov, ${ }^{1}$ A. P. Potylitsyn ${ }^{1,3}$, and G. Kube ${ }^{2,3}$ \\ 1) National Research Tomsk Polytechnic University, Tomsk, Russia; \\ 2) Deutsches Elektronen-Synchrotron (DESY), Hamburg, Germany; \\ 3) National Research Nuclear University MEPhI, Moscow, Russia.
}

In the majority of cases, modern systems for measuring the transverse profile of the electron beam in storage rings of synchrotron type work in the x-ray range in order to overcome the resolution limit imposed by diffraction. In conjunction with devices of this type, use is frequently made of a synchrotron radiation interferometer operating in the visible wavelength range. With the help of such an interferometer, consisting of a screen with two slits (1D interferometer), it is possible to measure the transverse dimension down to a few micrometers. $1 D$ interferometers are widely used in circular interferometer is used to determine the vertical dimension of the beam. It is proposed to use a $2 D$ interferometer, consisting of a screen with four circular holes, making it possible to measure the vertical and horizontal dimensions simultaneously.

Keywords: synchrotron radiation, beam dimensions, interferometer.

\section{INTRODUCTION}

A 1D synchrotron radiation interferometer for measuring the beam profile in a storage ring was first proposed by T. Mitsuhashi [1]. The principle of operation of a synchrotron radiation (SR) interferometer is based on measurement of the spatial coherence of the synchrotron radiation in the optical range. A diagram of the interferometer is shown in Fig. 1. Radiation emitted by the electron beam passes through an aperture mask, the lens, the bandpass filter, and the polarizer, after which an interference pattern is observed in the detector plane.

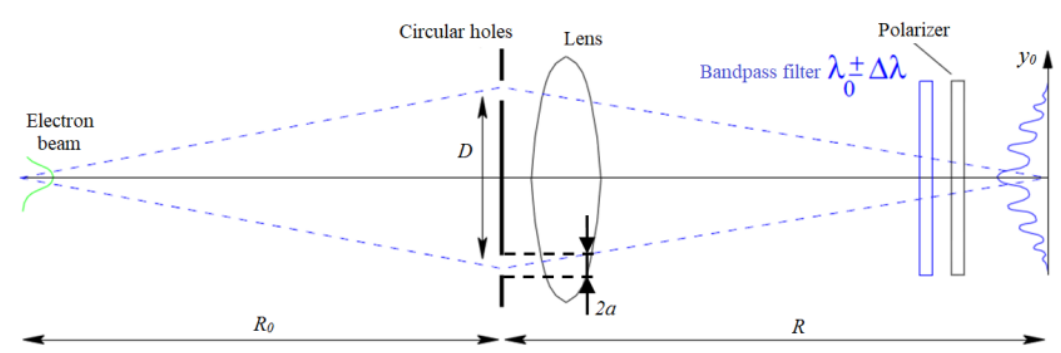

Fig. 1. Diagram of the interferometer.

Information about the size of the beam is determined from the depth of the interference minima: the smaller the beam size, the deeper the minima, and vice versa. Thus, if the source is a point source, the minima of the interference pattern will reach zero. This effect is illustrated in Fig. 2.

The intensity of the interference pattern depends on the value of the mutual spatial coherence $\gamma(D)$ [2] and in the case of circular apertures is given by the formula [3]

$$
I\left(y_{0}\right)=I_{0}\left[J_{1}\left(\frac{2 \pi a}{\lambda_{0} R} y_{0}\right) /\left(\frac{2 \pi a}{\lambda_{0} R} y_{0}\right)\right]^{2}\left[1+|\gamma(D)| \cos \left(\frac{2 \pi D}{\lambda_{0} R} y_{0}+\varphi\right)\right],
$$


where $a$ is the aperture radius, the distances $D$ and $R$ are shown in Fig. $1, \lambda_{0}$ is the wavelength of the observed SR, $I_{0}$ is the sum of the intensity of the SR from both gaps. Similarly, we introduce a formula for the case with rectangular gaps, which differs only in that trigonometric functions appear instead of $J_{1}$ [1]. If the Fraunhofer diffraction condition (distant wave zone) is met, then according to the Van Cittert-Zernike theorem $[4,5]$ the degree of coherence $\gamma(D)$ for a radiation source (electron beam), with transverse profile $f(y)$ - can be represented in the form of the Fourier transform

$$
\gamma(v)=\int f(y) \exp (-i 2 \pi v y) d y,
$$

where $v=\frac{D}{\lambda_{0} R}$ is a variable that depends on the inverse wave vector $\frac{2 \pi}{\lambda_{0}}$.

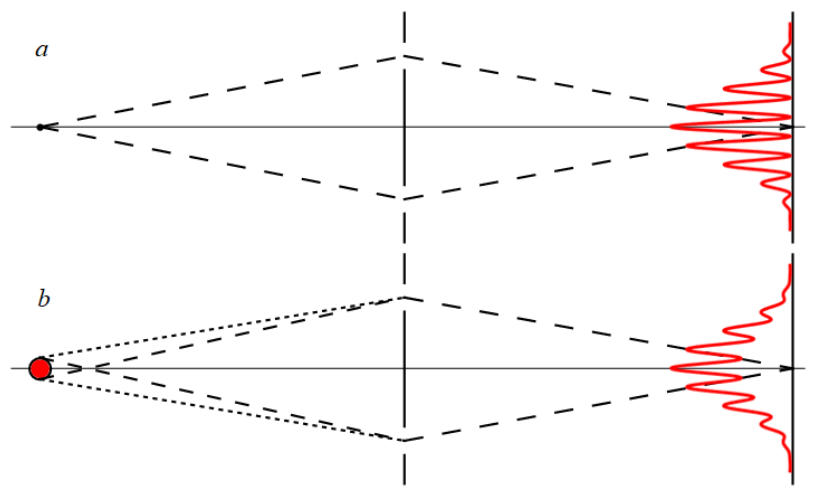

Fig. 2. Cases: $a$ ) point source of the synchrotron radiation, $b$ ) source with finite dimensions.

If the electron beam has a normal distribution, then its size can be calculated using the formula

$$
\sigma=\frac{\lambda_{0} R}{\pi D} \sqrt{\frac{1}{2} \ln \frac{1}{\gamma(D)}} .
$$

Thus, having obtained an interference pattern and knowing the parameters of the optical system, it is possible to calculate $\gamma(D)$ by approximating the interference pattern using formula (1) and then finding the transverse dimension of the beam, using formula (3).

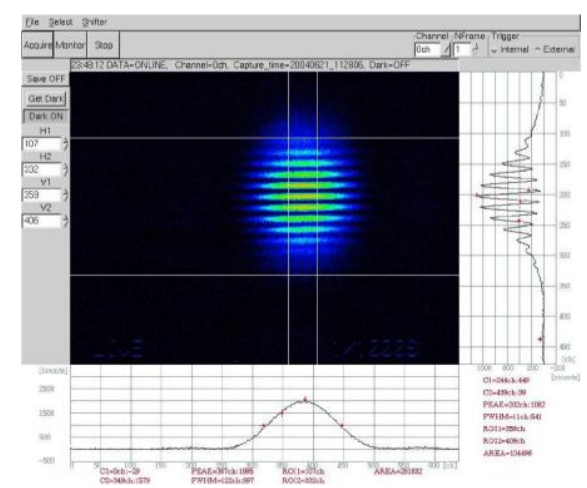

Fig. 3. Interference pattern of a 1D interferometer.

Figure 3 presents an interference pattern with transverse projections, taken on the 1D interferometer of the KEK storage ring (Japan) [6], which has been in use already for a few years. The white lines delineate the region from 
which the horizontal and vertical projections were taken. With the help of the given interferometer, only the vertical dimension of the beam can be determined.

\section{D SYNCHROTRON RADIATION INTERFEROMETER}

In this study, we propose to use the four apertures (holes) in the screen of the interferometer (2D interferometer) for the PETRA III accelerator. The design of the four-hole interferometer differs from the two-hole design only in the number and positions of the holes (Fig. 4) [7]. An interference pattern in the case of the 2D interferometer, modeled in the Synchrotron Radiation Workshop (SRW) program package [8], is presented in Fig. 5. The given program package allows one to write code, with the help of which the interferometer can be modeled for practically any geometry and shape of the apertures.
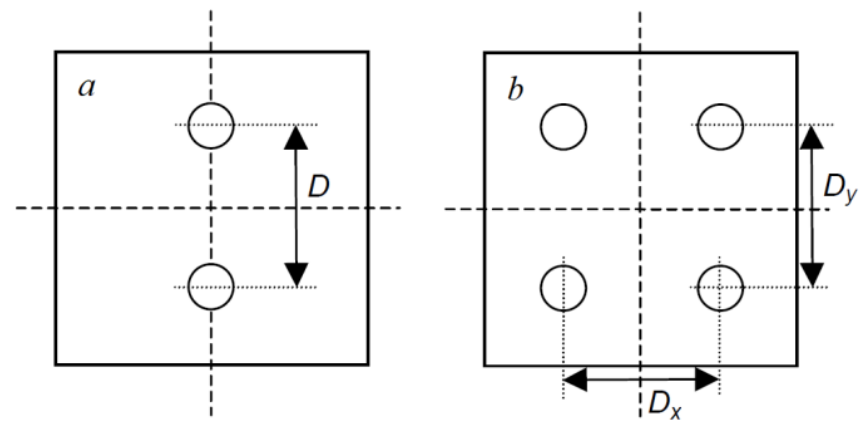

Fig. 4. Arrangement of the gaps: $a$ ) for the two-gap interferometer, $b$ ) for the four-gap interferometer

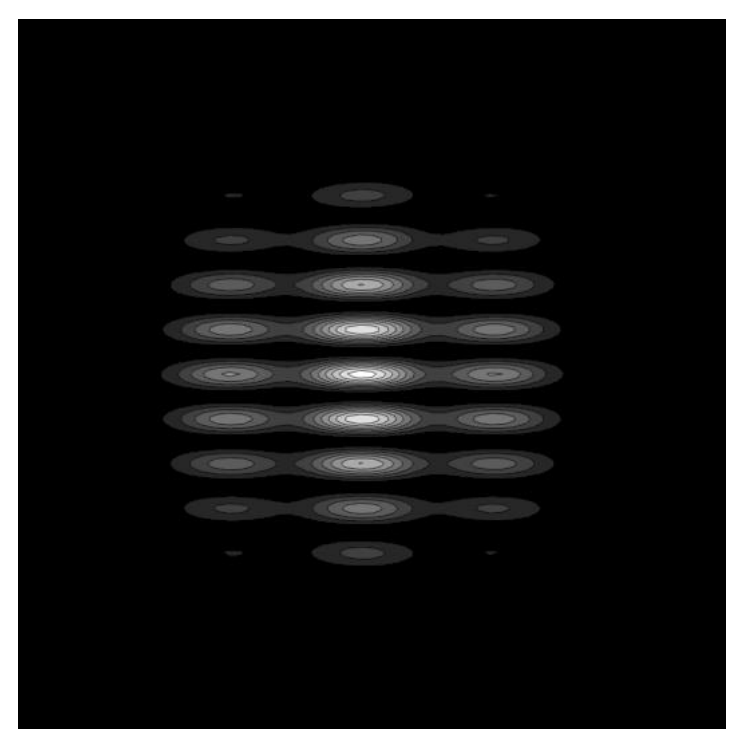

Fig. 5. Interference pattern of the 2D interferometer modeled in the program package SRW.

Thus, in the case of the 2D interferometer an interference pattern is observed in both the vertical and the horizontal direction. Consequently, both dimensions -_vertical and horizontal - can be reconstructed at once. The dimensions are reconstructed by fitting formula (1) to the horizontal and vertical projections with the distance between the holes in the corresponding direction $-D_{x}$ or $D_{y}$ - substituted for the parameter $D$ in the formula. Consequently, formula (1) for the horizontal and vertical directions will look like this: 


$$
\begin{aligned}
& I(x)=I_{0}\left[J_{1}\left(\frac{2 \pi a}{\lambda_{0} R} x\right) /\left(\frac{2 \pi a}{\lambda_{0} R} x\right)\right]^{2}\left[1+\left|\gamma\left(D_{x}\right)\right| \cos \left(\frac{2 \pi D_{x}}{\lambda_{0} R} x+\varphi\right)\right], \\
& I(y)=I_{0}\left[J_{1}\left(\frac{2 \pi a}{\lambda_{0} R} y\right) /\left(\frac{2 \pi a}{\lambda_{0} R} y\right)\right]^{2}\left[1+\left|\gamma\left(D_{y}\right)\right| \cos \left(\frac{2 \pi D_{y}}{\lambda_{0} R} y+\varphi\right)\right] .
\end{aligned}
$$

It is important to select the parameters $D$ and $a$, even if only roughly, at the modeling stage. These parameters determine the number of peaks and the width of the interference pattern. To demonstrate, we modeled some interference patterns in the vertical direction for arbitrary values of $I_{0}, \lambda_{0}, R$, and $\gamma\left(D_{y}\right)$ while varying the values of $a$ and $D_{y}$. The results are shown in Figs. 6 and 7.

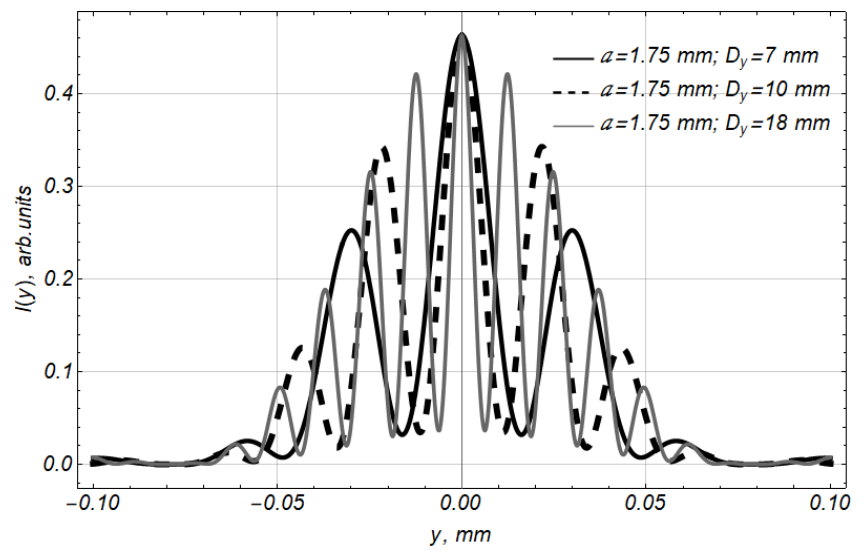

Fig. 6. Interference pattern in the vertical direction for different values of the parameter $D$.

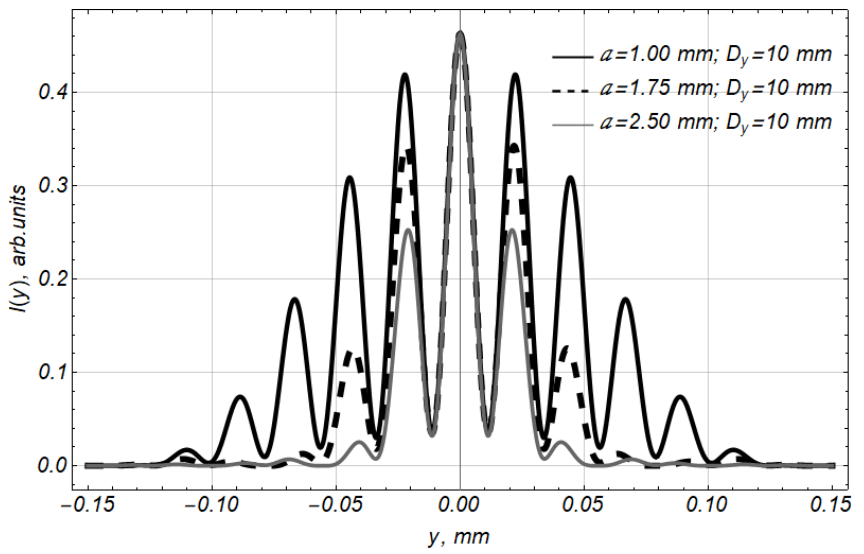

Fig. 7. Interference pattern in the vertical direction for different values of the parameter $a$.

In Fig. 6 it can be seen that with increase of $D$ the overall width of the interference pattern is preserved whereas the number of peaks grows, while in Fig. 7 it can be seen that the number of peaks grows on account of an increase in the overall width of the interference pattern. Knowing the wavelength that we will use in the measurements $\left(\lambda_{0}\right)$, the distance from the gaps to the detector $(R)$, and the rough dimensions of the beam in both directions, we can model the interferometer and select rough optimal dimensions $D_{x}, D_{y}$, and $a$, so that the dimensions and number of the peaks of the interference pattern will match the dimensions and resolution of the detector. In the given case, such modeling was performed for the parameters of the PETRA III accelerator.

The PETRA III bending magnet in use for the interferometer [9] (electron energy $E_{e}=6.01 \mathrm{GeV}$, orbit radius $R_{\text {orb }}=191.73 \mathrm{~m}$, and average beam current $I \sim 100 \mathrm{~mA}$ ) generates an SR beam in the x-ray range with characteristic 
photon energy $\hbar \omega_{S R}=1.5 \gamma^{3} \hbar c / R_{\text {orb }}=2.5 \mathrm{keV}$ (here $\gamma=E_{e} / m c^{2}=1.18 \cdot 10^{4}$ ) [9]. The photon yield per second in the plane perpendicular to the orbit is found from the well-known single electron spectral-angular distribution of the SR power [10]:

$$
\frac{d P}{d \omega d \psi}=\frac{3}{4 \pi^{2}} \alpha \frac{\hbar c}{R}\left(\frac{\omega}{\omega_{c}}\right)^{2} \gamma^{2}\left(1+\gamma^{2} \psi^{2}\right)^{2}\left[K_{2 / 3}^{2}(\xi)+\frac{\gamma^{2} \psi^{2}}{1+\gamma^{2} \psi^{2}} K_{1 / 3}^{2}(\xi)\right]
$$

where $\psi$ is the vertical angle, $\omega_{c}$ is the characteristic frequency of the spectrum, $\xi=\frac{1}{2} \frac{\omega}{\omega_{c}}\left[1+\gamma^{2} \psi^{2}\right]^{3 / 2}$, and $K_{1 / 3}(\xi)$ and $K_{2 / 3}(\xi)$ are modified Bessel functions.

For the proper operation of the interferometer it was necessary to estimate the photon yield for an optical bandpass filter with central wavelength $\lambda_{0}=450 \mathrm{~nm}$ and bandwidth $\Delta \lambda_{0}=10 \mathrm{~nm}$. The dependence of the SR intensity on the vertical angle (in the plane perpendicular to the orbit) is plotted in Fig. 8 for $\lambda_{0}=450 \mathrm{~nm}$. For the aperture diameter $2 a$ the angular spread can be estimated as $\Delta \psi=2 a / R_{0}$ (shown by the dashed lines for $a=1 \mathrm{~mm}$ and $\left.R_{0}=14.2 \mathrm{~m}\right)$. Thus, the efficiency of the vertical aperture $f_{\psi}$ can be estimated as

$$
f_{\psi} \approx \int_{(D-4 a) / 2 R_{0}}^{(D+4 a) / 2 R_{0}} \frac{d P}{d \omega d \psi} d \psi / \int_{0}^{\pi} \frac{d P}{d \omega d \psi} d \psi \approx 0.13
$$

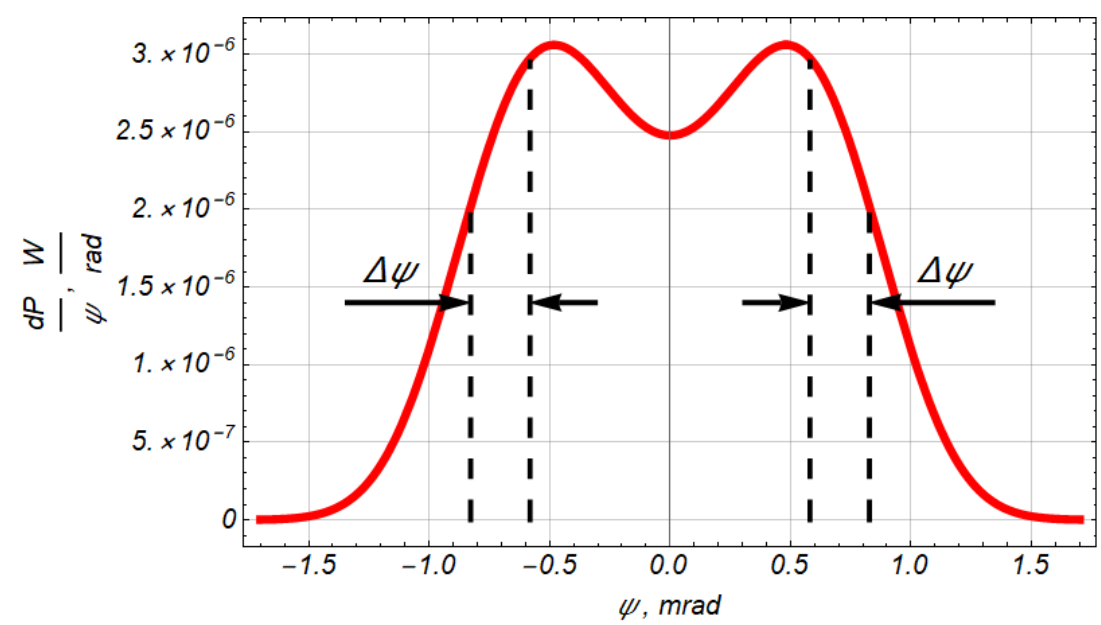

Fig. 8. Schematic depiction of the optical system of the interferometer.

The intensity of the SR photons emitted per second per unit solid angle in the energy range $\Delta \omega_{0} / \omega_{0}=0.1 \%$ is given by the well-known formula [11]

$$
I\left[\mathrm{phot} /\left(\mathrm{s} \cdot \operatorname{mrad}^{2}\right) 0.1 \% b w\right]=2.46 \cdot 10^{10} I[\mathrm{~mA}] E_{e}[\mathrm{GeV}] G_{1}\left(\omega / \omega_{S R}\right)
$$

Here, the function $G_{1}\left(\omega_{1} / \omega_{S R}\right)$ is given by the expression

$$
G_{1}\left(\omega / \omega_{S R}\right)=\frac{\omega}{\omega_{S R}} \int_{\omega / \omega_{S R}}^{\infty} K_{5 / 3} d y
$$


For $\omega / \omega_{S R}=1.1 \cdot 10^{-3} G_{1}\left(\omega / \omega_{S R}\right) \sim 0.2$.

The photon yield per second into the assigned aperture is estimated from formulas (4) and (5):

$$
\Delta N_{\phi} \approx I \Delta \Omega \approx I\left(\frac{\pi a^{2}}{R_{0}^{2}}\right) .
$$

For the parameters of the PETRA III storage-ring accelerator and for $a=1 \mathrm{~mm}$ and $R_{0}=14.2 \mathrm{~m}$, we obtain the estimate

$$
\Delta N_{\phi} \sim 10^{4} \mathrm{phot} / \mathrm{s}
$$

allowing us to choose the exposure time for simultaneous measurement of the parameters $\sigma_{x}$ and $\sigma_{y}$.

\section{D-INTERFEROMETER OF THE PETRA III ACCELERATOR}

Figure 9 schematically depicts the optical system of the 2D-interferometer of the PETRA III storage-ring. Radiation, generated by the electron beam, is reflected by mirrors 1 and 2 out of the accelerator tunnel, after which it is directed by mirror 3 into the optical system consisting of the aperture mask, focusing lens 1 (Borg $50 \mathrm{ED}, F=510 \mathrm{~mm}$ ), optical bandpass filter $\left(\lambda_{0}=450 \mathrm{~nm}, \mathrm{FWHM}=10 \mathrm{~nm}\right)$, magnifying lens, and polarizer (Karl Lambrecht Corporation, MGT25S20) to extract the $\sigma$-polarization. It is then recorded by the CCD camera (JAI BM-141GE). On the basis of our modeling results, we chose the following geometry for the aperture mask (see Fig. 4): $D_{x}=20 \mathrm{~mm}, D_{y}=7 \mathrm{~mm}$, and $a=1.75 \mathrm{~mm}$.

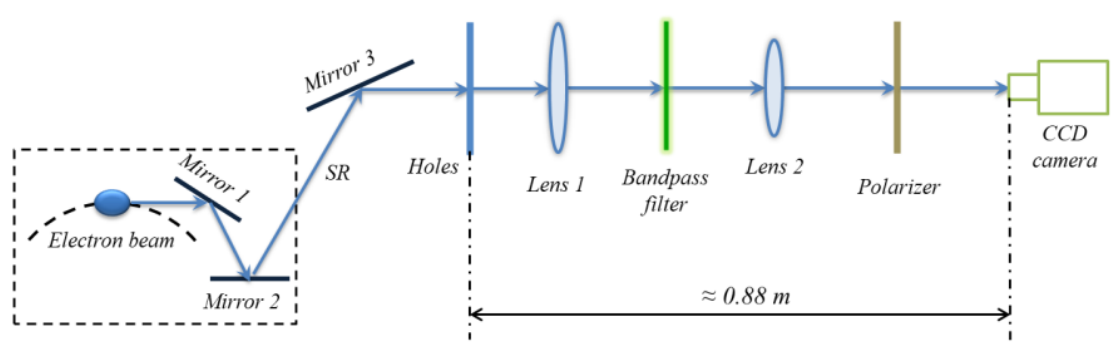

Fig. 9. Schematic depiction of the optical system of the interferometer.

Figure 10 depicts one of the first interference patterns obtained on the 2D interferometer of the PETRA III accelerator, and also its horizontal and vertical projections.

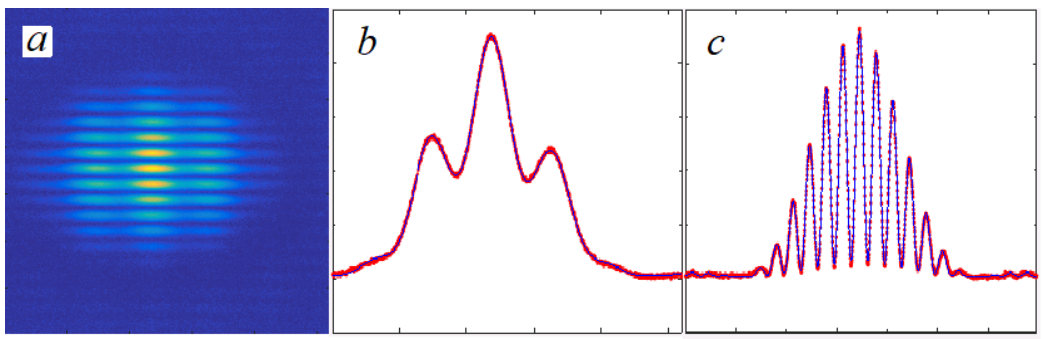

Fig. 10. One of the first interference patterns obtained on the 2D interferometer $(a)$, horizontal projection $(b)$, and vertical projection $(c)$. 


\section{CONCLUSIONS}

In this paper we have presented modeling results for the 2D interferometer of the PETRA III storage-ring accelerator and sketched out its subsequent development. We have shown that such an interferometer allows one to measure the dimensions $\sigma_{x}$ and $\sigma_{y}$. A code has been written that allows one to model a 2D interferometer with an aperture mask of arbitrary shape and geometry of their arrangement in accordance with the requirements of the experiment.

One of the authors (A. I. Novokshonov) expresses his gratitude to the DESY research center for financial support and the possibility of carrying out experiments.

This work was performed with the partial support of the Russian Ministry of Education and Science Project No. 3.1903.2017/ПЧ.

\section{REFERENCES}

1. T. Mitsuhashi, Proc. of the Joint US - CERN - Japan - Russia School on Particle Accelerators, Montreux (1998), pp. 399-427.

2. M. Born and E. Wolf, Principles of Optics, Pergamon Press, New York (1980).

3. L. Torino and U. Iriso, Proc. of IBIC'15, Melbourne, Australia (2015).

4. P. H. Van Cittert, Physica, 1, 201 (1934).

5. F. Zernike, Physica, 5, 785 (1938).

6. M. Katoh and T. Mitsuhashi, Proc. of PAC'99, New York, USA (1999), p. 2307.

7. M. Masaki and S. Takano, Proc. of DIPAC'01, Grenoble, France (2001), p. 142.

8. https://github.com/ochubar/SRW - Developer's Page - Synchrotron Radiation Workshop.

9. http://photon-science.desy.de/facilities/petra_iii/machine/parameters/index_eng.html - page with parameters of the PETRA III accelerator.

10. V. G. Bagrov and V. A. Bordovitsyn, Theory of the Radiation of Relativistic Particles [in Russian], Fizmatlit, Moscow (2002).

11. L. Rivkin, CERN Accelerator School: Synchrotron Radiation Lecture, Varna, Bulgaria (2010). 